University of New Orleans

ScholarWorks@UNO

10-30-2008

\title{
Plurality of principal angles for a given pseudo-Brewster angle when polarized light is reflected at a dielectric-conductor interface
}

R. M.A. Azzam

University of New Orleans, razzam@uno.edu

A. Alsamman

University of New Orleans, aalsamma@uno.edu

Follow this and additional works at: https://scholarworks.uno.edu/ee_facpubs

Part of the Electrical and Electronics Commons, and the Optics Commons

\section{Recommended Citation}

R. M. A. Azzam and A. Alsamman, "Plurality of principal angles for a given pseudo-Brewster angle when polarized light is reflected at a dielectric-conductor interface," J. Opt. Soc. Am. A 25, 2858-2864 (2008)

This Article is brought to you for free and open access by the Department of Electrical Engineering at ScholarWorks@UNO. It has been accepted for inclusion in Electrical Engineering Faculty Publications by an authorized administrator of ScholarWorks@UNO. For more information, please contact scholarworks@uno.edu. 


\title{
Plurality of principal angles for a given pseudo-Brewster angle when polarized light is reflected at a dielectric-conductor interface
}

\author{
R. M. A. Azzam* and A. Alsamman \\ Department of Electrical Engineering, University of New Orleans, New Orleans, Louisiana 70148, USA \\ *Corresponding author: razzam@uno.edu
}

Received July 17, 2008; accepted September 12, 2008;

posted September 22, 2008 (Doc. ID 98934); published October 30, 2008

\begin{abstract}
The pseudo-Brewster angle $\phi_{p B}$ of minimum reflectance for $p$-polarized light and the principal angle $\bar{\phi}$ at which incident linearly polarized light of the proper azimuth is reflected circularly polarized are considered as functions of the complex relative dielectric function $\varepsilon$ of a dielectric-conductor interface over the entire complex $\varepsilon$ plane. In particular, the spread of $\bar{\phi}$ for a given $\phi_{p B}$ is determined, and the maximum difference $\left(\bar{\phi}-\phi_{p B}\right)_{\max }$ is obtained as a function of $\phi_{p B}$. The maximum difference $\left(\bar{\phi}-\phi_{p B}\right)_{\max }$ approaches $45^{\circ}$ and 0 in the limit as $\phi_{p B} \rightarrow 0$ and $90^{\circ}$, respectively. For $\phi_{p B}<22.666^{\circ}$, multiple principal angles $\bar{\phi}_{i}, i=1,2,3$, appear for each $\varepsilon$ in a subdomain of fractional optical constants. This leads to an elaborate pattern of multiple solution branches for the difference $\bar{\phi}_{i}-\phi_{p B}, i=1,2,3$, as is illustrated by several examples. () 2008 Optical Society of America

OCIS codes: $120.5700,240.0240,240.2130,260.0260,260.3910,260.5430$.
\end{abstract}

\section{INTRODUCTION}

The reflection of monochromatic $p$ - and $s$-polarized light at an angle $\phi$ by the planar interface between a transparent medium of incidence (ambient) of refractive index $n_{0}$ and an absorbing medium of refraction (substrate) of complex refractive index $N_{1}=n_{1}-j k_{1}$ is governed by the wellknown complex-amplitude Fresnel reflection coefficients [1-3]:

$$
\begin{aligned}
r_{p} & =\frac{\varepsilon \cos \phi-\left(\varepsilon-\sin ^{2} \phi\right)^{1 / 2}}{\varepsilon \cos \phi+\left(\varepsilon-\sin ^{2} \phi\right)^{1 / 2}}, \\
r_{s} & =\frac{\cos \phi-\left(\varepsilon-\sin ^{2} \phi\right)^{1 / 2}}{\cos \phi+\left(\varepsilon-\sin ^{2} \phi\right)^{1 / 2}}, \\
\varepsilon & =N_{1}{ }^{2} / n_{0}{ }^{2}=(n-j k)^{2}=\varepsilon_{r}-j \varepsilon_{i} .
\end{aligned}
$$

For a given value of the complex relative dielectric function $\varepsilon$, which is characteristic of a given interface at a given wavelength, the amplitude reflectance $\left|r_{p}\right|$ of $p$-polarized light as a function of $\phi$ reaches a minimum at the pseudo-Brewster angle (PBA) $\phi_{p B}$. If the medium of refraction is also transparent, $\varepsilon_{i}=0$, the minimum reflectance is zero, $\left|r_{p}\right|_{\min }=0$, and the PBA $\phi_{p B}$ reverts back to the usual Brewster angle $\phi_{B}=\tan ^{-1} \sqrt{\varepsilon_{r}}$. Recall that for any $\varepsilon$ the amplitude reflectance $\left|r_{s}\right|$ of $s$-polarized light increases monotonically as a function of $\phi$ between normal and grazing incidence, $0 \leqslant \phi \leqslant 90^{\circ}$.

The first correct derivation of the relation between $\phi_{p B}$ and complex $\varepsilon$ (which replaces Brewster's law) is believed to be that of Humphreys-Owen [4], as was noted by Holl [5]. Continued interest in this salient feature of the reflec- tion of $p$-polarized light (and other electromagnetic waves) at a dielectric-conductor interface has led to several subsequent derivations [6-9].

Another important and distinct angle of incidence is the principal angle (PA) $\bar{\phi}$ at which incident linearly polarized light of the proper azimuth (called the principal azimuth $\bar{\psi}$ ) is reflected circularly polarized [1-3,10]. This occurs when the differential reflection phase shift $\Delta$ of $p$ and $s$-polarized light is quarter-wave, i.e.,

$$
\begin{aligned}
& \Delta=\delta_{p}-\delta_{s}=90^{\circ}, \\
& \delta_{p}=\arg \left(r_{p}\right), \quad \delta_{s}=\arg \left(r_{s}\right) .
\end{aligned}
$$

The ratio of complex $p$ and $s$ reflection coefficients, also known as the ellipsometric function $\rho$ [2], is obtained from Eqs. (1) and (2) as

$$
\rho=r_{p} / r_{s}=\frac{\sin \phi \tan \phi-\left(\varepsilon-\sin ^{2} \phi\right)^{1 / 2}}{\sin \phi \tan \phi+\left(\varepsilon-\sin ^{2} \phi\right)^{1 / 2}} .
$$

At the principal angle, $\phi=\bar{\phi}, \rho$ becomes pure imaginary,

$$
\rho=\bar{\rho}=j \tan \bar{\psi}
$$

For a given complex $\varepsilon$, the PA, $\bar{\phi}$, is determined by solving a cubic equation [10]:

$$
\begin{gathered}
a_{3} u^{3}+a_{2} u^{2}+a_{1} u+a_{0}=0, \\
a_{0}=\varepsilon_{r}^{2}+\varepsilon_{i}^{2}, \quad a_{1}=-2\left(a_{0}+\varepsilon_{r}\right), \\
a_{2}=a_{0}+4 \varepsilon_{r}+1, \quad a_{3}=-2\left(\varepsilon_{r}+1\right),
\end{gathered}
$$




$$
u=\sin ^{2} \bar{\phi}
$$

Over much of the complex plane, Eqs. (7)-(9) yield only one acceptable root $(0<u<1)$, hence one PA $\bar{\phi}$, for each $\varepsilon$. However, as has been noted in [5,10], there exists a small but important region of fractional optical constants $(0$ $\left.<\left|\varepsilon_{r}\right|,\left|\varepsilon_{i}\right|<1\right)$ within which three distinct PAs exist for each complex $\varepsilon$. This domain of multiple principal angles (MPAs), shown highlighted in Fig. 1, is bounded by the real axis, $\varepsilon_{i}=0$, and the curve whose parametric equation is given by [10]

$$
\begin{gathered}
\varepsilon_{r}=u+\frac{u^{3}(u-2)}{(1-u)^{3}}, \\
\varepsilon_{i}=\frac{\left(2 u^{6}-4 u^{5}+u^{4}\right)^{1 / 2}}{(1-u)^{3}}, \\
0 \leqslant u \leqslant 1-\frac{1}{\sqrt{2}}=0.293 .
\end{gathered}
$$

Equations (10) represent the locus of all possible values of complex $\varepsilon$ for which two of the three principal angles, $\bar{\phi}$ $=\sin ^{-1} \sqrt{u}$, coincide; this locus is represented by the dashed curve in Fig. 1 . The cusp point $P$ corresponds to $u=1 / 4$ and is located at $\varepsilon=(5 / 27, \sqrt{2} / 27)$. Fractional optical constants are encountered for many materials in the vacuum UV and x-ray spectral regions $[11,12]$ and also in attenuated or total internal reflection when light is incident from an optically dense medium [13].

Because of approximate formulations used in metal optics, the PBA and PA are sometimes erroneously presumed to be the same. In this paper the difference between these two angles, $\bar{\phi}-\phi_{p B}$, is thoroughly investigated as a function of complex $\varepsilon$. This is accomplished in Section 2 by deliberately holding $\phi_{p B}$ constant and determining all possible values of the associated PA $\bar{\phi}$. The maximum difference $\left(\bar{\phi}-\phi_{p B}\right)_{\max }$ is also determined as a function of $\phi_{p B}$. Unusual results are obtained in the domain of MPAs, as is described in Section 3. Finally, Section 4 gives a brief summary of the paper.

\section{RANGE OF PRINCIPAL ANGLES FOR A GIVEN PSEUDO-BREWSTER ANGLE}

All possible values of complex $\varepsilon=\left(\varepsilon_{r}, \varepsilon_{i}\right)$ for which the PBA $\phi_{p B}$ is one and the same are obtained as follows [14]:

$$
\begin{gathered}
\varepsilon_{r}=|\varepsilon| \cos \theta, \quad \varepsilon_{i}=|\varepsilon| \sin \theta, \\
|\varepsilon|=\ell \cos (\zeta / 3), \\
\ell=\frac{2 u[1-(2 u / 3)]^{1 / 2}}{(1-u)}, \\
\zeta=\cos ^{-1}\left(-\frac{(1-u) \cos \theta}{[1-(2 u / 3)]^{3 / 2}}\right), \\
u=\sin ^{2} \phi_{p B}, \quad 0 \leqslant \theta \leqslant 180^{\circ} .
\end{gathered}
$$

As $\theta$ is increased from 0 to $180^{\circ}$, the minimum reflectance $\left|r_{p}\right|_{\text {min }}$ at the same $\phi_{p B}$ increases monotonically from 0 to 1 [15]. For given $\phi_{p B}$, and for each $\theta$ from 0 to $180^{\circ}$ in steps of $1^{\circ} \varepsilon=\left(\varepsilon_{r}, \varepsilon_{i}\right)$ is calculated using Eqs. (11) and (12) and the corresponding values of $\bar{\phi}$ are determined from Eqs. (7)-(9).

In Fig. 2 the difference $\bar{\phi}-\phi_{p B}$ is plotted as a function of $\theta, 0 \leqslant \theta \leqslant 180^{\circ}$, for constant values of $\phi_{p B}$ from $25^{\circ}$ to $85^{\circ}$ in equal steps of $5^{\circ}$. For each $\phi_{p B}$ in this range, there is only one PA, $\bar{\phi}>\phi_{p B}$, and the difference $\bar{\phi}-\phi_{p B}$ increases monotonically as a function of $\theta$. In Fig. 2 the curve for $\phi_{p B}=85^{\circ}$ almost coincides with the $\theta$ axis.

From Fig. 2 it is also apparent that the maximum difference $\left(\bar{\phi}-\phi_{p B}\right)_{\max }$ occurs at $\theta=180^{\circ}$ and that

$$
\partial\left(\bar{\phi}-\phi_{p B}\right) / \partial \theta=0, \quad \theta=0,180^{\circ}
$$

At the limiting angle $\theta=180^{\circ}$, Eqs. (11) and (12) yield $\varepsilon_{i}$ $=0$ and $\varepsilon_{r}<0$ given by

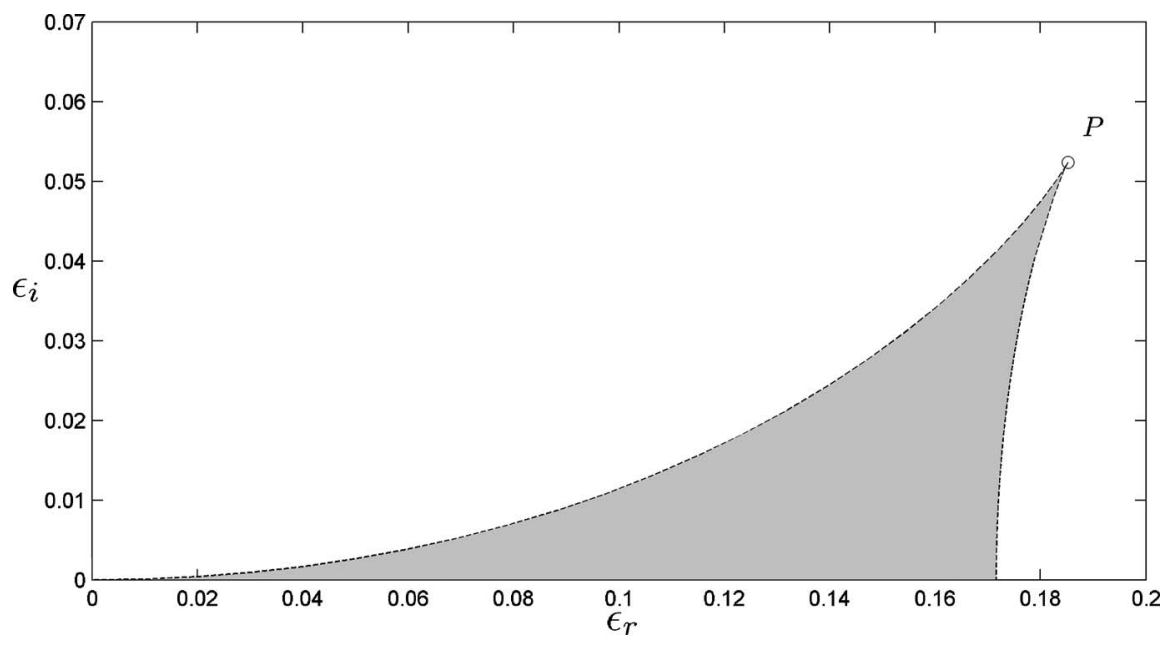

Fig. 1. Domain of MPAs, shown highlighted, is bounded by the real axis, $\varepsilon_{i}=0$, and the dashed curve described by Eqs. [10]. Cusp point $P$ is located at $\varepsilon=(5 / 27, \sqrt{2} / 27)=(0.1852,0.0524)$. 


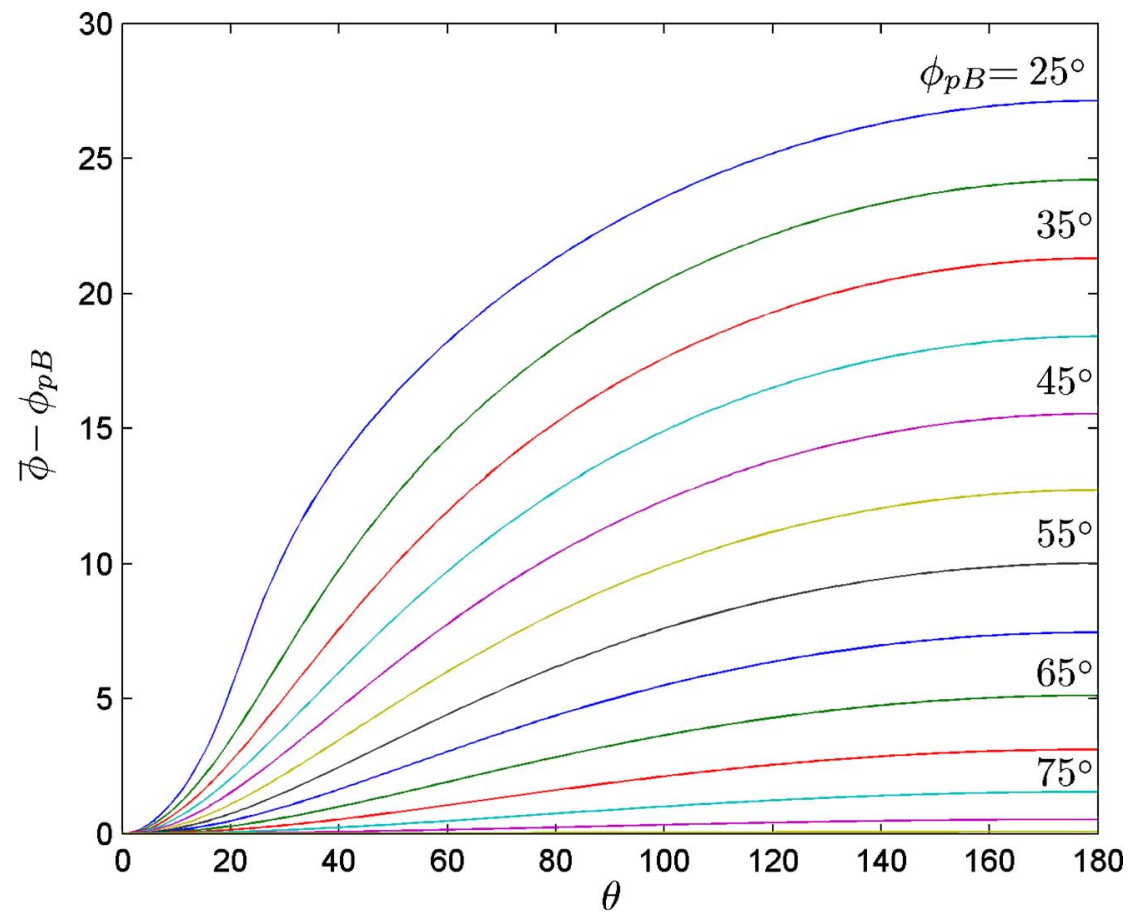

Fig. 2. (Color online) Difference of PA and PBA $\bar{\phi}-\phi_{p B}$ plotted as a function of the angle $\theta$ of complex $\varepsilon, 0 \leqslant \theta \leqslant 180^{\circ}$, for constant values of $\phi_{p B}$ from $25^{\circ}$ to $85^{\circ}$ in equal steps of $5^{\circ}$.

$$
\varepsilon=\varepsilon_{r}=-\frac{1}{2} \tan ^{2} \phi_{p B}\left[1+\left(9-8 \sin ^{2} \phi_{p B}\right)^{1 / 2}\right] .
$$

The maximum PA $\bar{\phi}$ that corresponds to $\varepsilon_{r}$ of Eq. (14) is given by

$$
\bar{\phi}_{\max }=\sin ^{-1}\left\{\frac{1}{2}\left[\left(\varepsilon_{r}+1\right)+\left(\varepsilon_{r}^{2}-6 \varepsilon_{r}+1\right)^{1 / 2}\right]^{1 / 2}\right\} .
$$

The maximum difference $\left(\bar{\phi}-\phi_{p B}\right)_{\max }$ calculated from Eqs. (14) and (15) is $24.207^{\circ}, 15.540^{\circ}, 7.458^{\circ}$, and $0.073^{\circ}$ when $\phi_{p B}=30^{\circ}, 45^{\circ}, 60^{\circ}$, and $85^{\circ}$, respectively. Figure 3

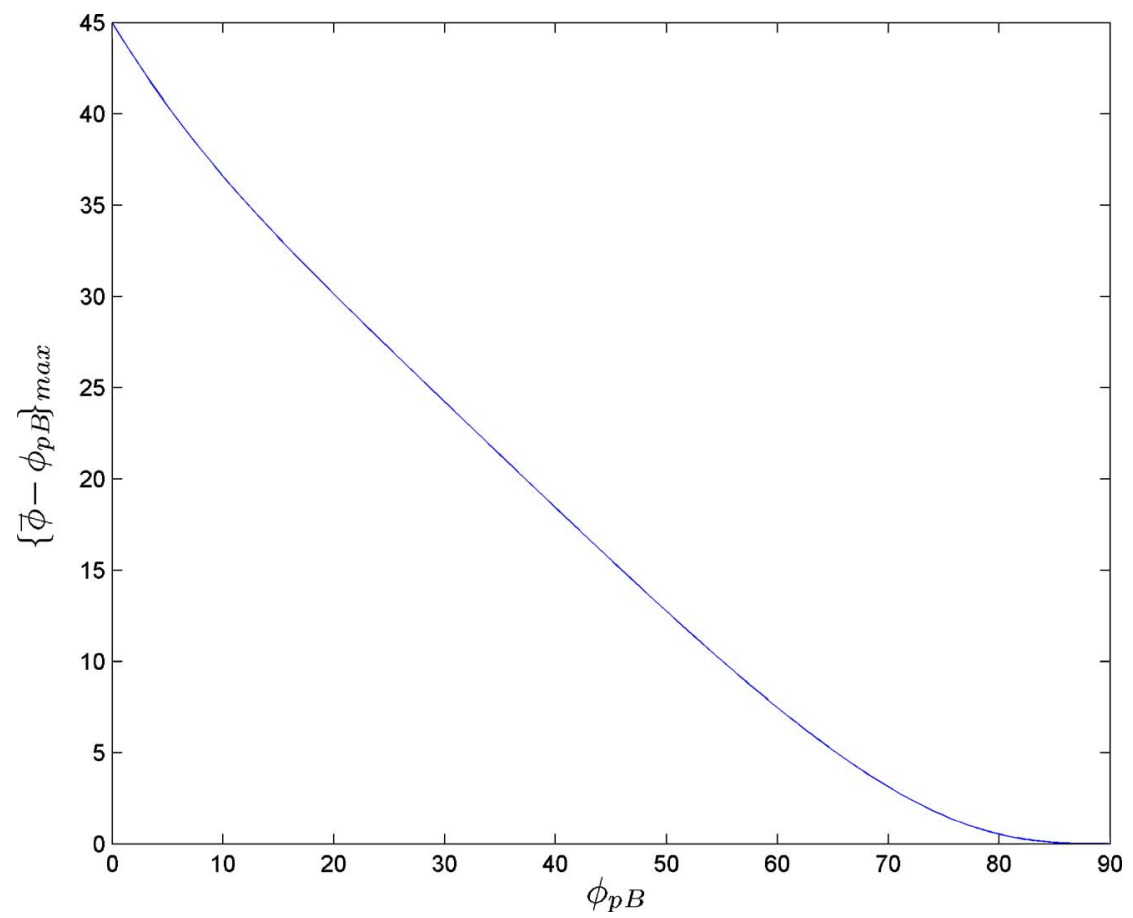

Fig. 3. (Color online) Maximum difference $\left(\bar{\phi}-\phi_{p B}\right)_{\max }$ as a function of $\phi_{p B}$ over the entire range $0<\phi_{p B}<90^{\circ}$. 


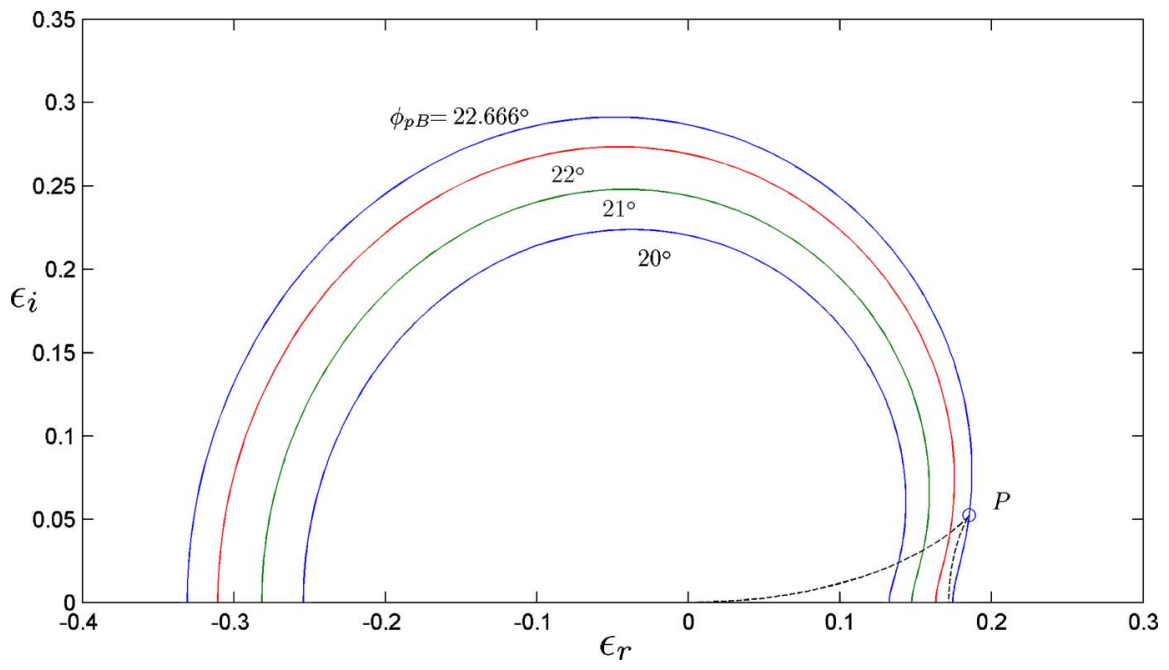

Fig. 4. (Color online) Constant-pseudo-Brewster-angle contour (CPBAC) in the complex $\varepsilon$ plane that corresponds to $\phi_{p B}=20^{\circ}, 21^{\circ}, 22^{\circ}$, and $22.666^{\circ}$. The CPBAC at $\phi_{p B}=22.666^{\circ}$ passes through the cusp point $P$.

shows $\left(\bar{\phi}-\phi_{p B}\right)_{\max }$ plotted versus $\phi_{p B}$ over the entire range $0<\phi_{p B}<90^{\circ}$. Notice that $\left(\bar{\phi}-\phi_{p B}\right)_{\max }=45^{\circ}$ in the limit as $\phi_{p B} \rightarrow 0$ and that $\left(\bar{\phi}-\phi_{p B}\right)_{\max }=0$ in the limit as $\phi_{p B} \rightarrow 90^{\circ}$. The latter limit is approached by metals in the far IR [9].

\section{DOMAIN OF MULTIPLE PRINCIPAL ANGLES}

MPAs exist when the PBA falls in the range

$$
0<\phi_{p B}<22.666^{\circ}
$$

Figure 4 shows four constant-PBA contours (CPBAC) in the complex $\varepsilon$ plane that correspond to $\phi_{p B}=20^{\circ}, 21^{\circ}, 22^{\circ}$, and $22.666^{\circ}$. The CPBAC at $\phi_{p B}=22.666^{\circ}$ passes through the cusp point $P$ on the boundary curve of the domain of MPAs (Fig. 1). The squared sine of this particular angle $\left(\phi_{p B}=22.666^{\circ}\right)$ satisfies the following cubic equation [14]:

$$
324 u^{3}-80 u^{2}-2 u+1=0 .
$$

As an example of MPAs, consider $\varepsilon=(0.1349,0.0118)$, which corresponds to $\theta=5^{\circ}$ on the CPBAC $\phi_{p B}=20^{\circ}$. For this value of complex $\varepsilon$, Fig. 5 shows $\left|r_{p}\right|,\left|r_{s}\right|$, and $\Delta$ as functions of the angle of incidence $\phi$. The minimum reflec-

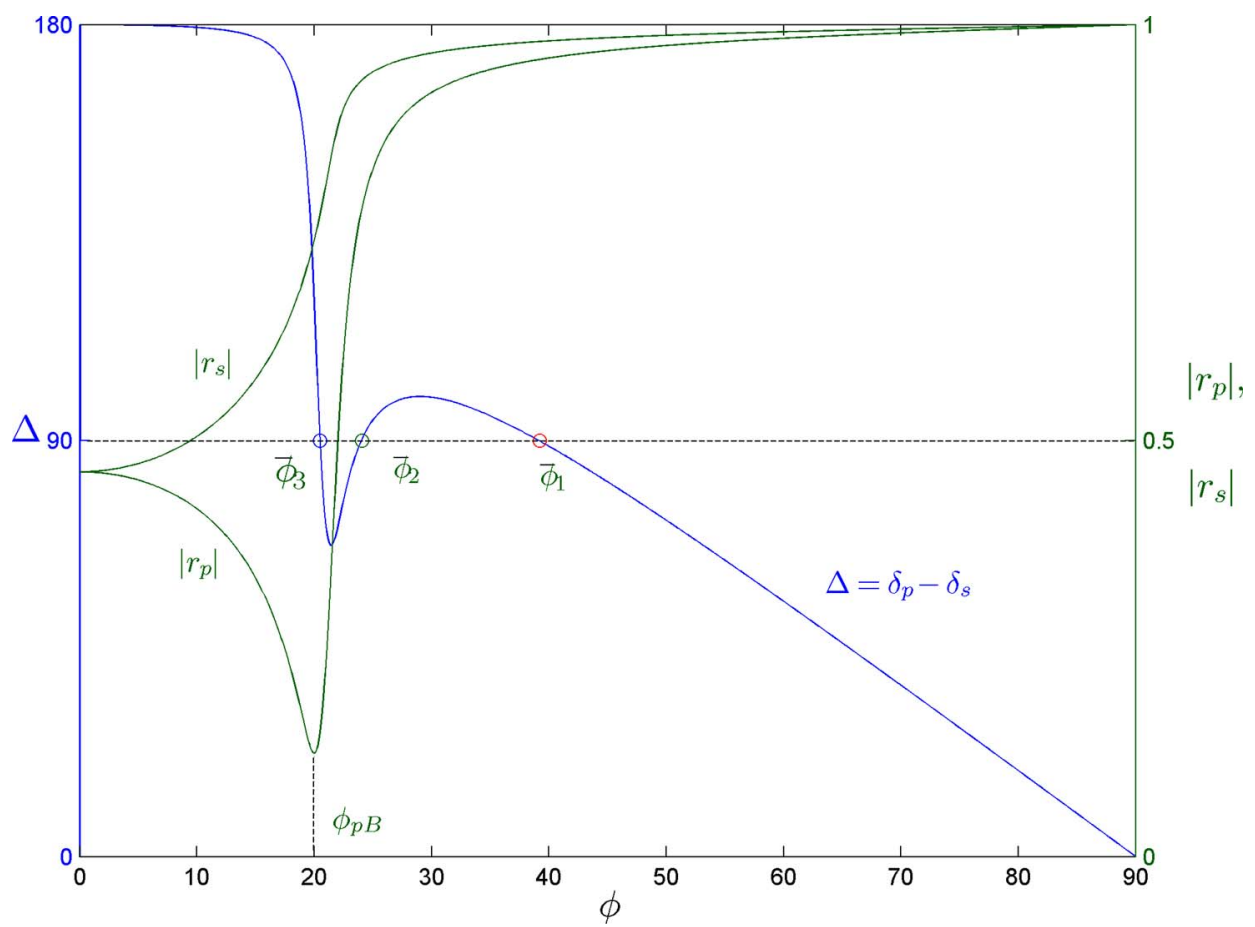

Fig. 5. (Color online) Amplitude reflectances $\left|r_{p}\right|,\left|r_{s}\right|$ and differential reflection phase shift $\Delta$ plotted as functions of the angle of incidence $\phi$ when $\varepsilon=(0.1349,0.0118)$. Minimum reflectance $\left|r_{p}\right|_{\min }$ is located at $\phi=\phi_{p B}=20^{\circ}$, and $\Delta=90^{\circ}$ occurs at three distinct PAs: $\bar{\phi}_{1}$ $=39.13^{\circ}, \bar{\phi}_{2}=24.01^{\circ}$, and $\bar{\phi}_{3}=20.49^{\circ}$. 


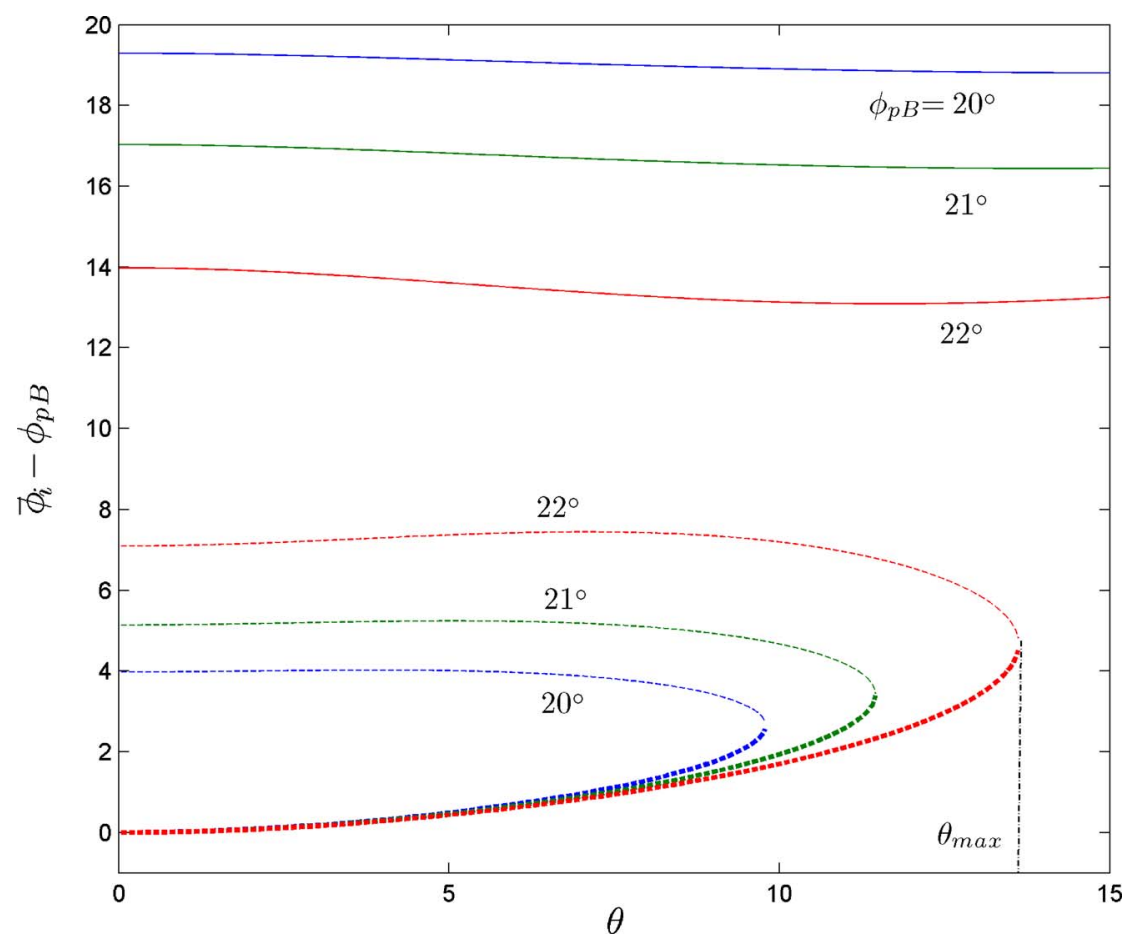

Fig. 6. (Color online) Multiple solution branches of the difference function $\bar{\phi}_{i}-\phi_{p B}, i=1,2,3$, plotted versus the angle $\theta$ of complex $\varepsilon$, for $\phi_{p B}=20^{\circ}, 21^{\circ}$, and $22^{\circ}$. For each $\phi_{p B}$ the solid, thin-dashed, and thick-dashed curves correspond to $\bar{\phi}_{1}>\bar{\phi}_{2}>\bar{\phi}_{3}$.

tance $\left|r_{p}\right|_{\min }$ appears at $\phi=\phi_{p B}=20^{\circ}$, and $\Delta=90^{\circ}$ occurs at three distinct PAs: $\bar{\phi}_{1}=39.13^{\circ}, \bar{\phi}_{2}=24.01^{\circ}$, and $\bar{\phi}_{3}$ $=20.49^{\circ}$. All three PAs $\bar{\phi}_{i}, i=1,2,3$, are $>\phi_{p B}$, which is true for any complex $\varepsilon$.

Figure 6 shows multiple solution branches $\bar{\phi}_{i}-\phi_{p B}, i$ $=1,2,3$, as functions of $\theta$ for $\phi_{p B}=20^{\circ}, 21^{\circ}$, and $22^{\circ}$. For each $\phi_{p B}$ the solid, thin-dashed, and thick-dashed curves correspond to $i=1,2,3$, respectively, where $\bar{\phi}_{1}>\bar{\phi}_{2}>\bar{\phi}_{3}$. MPAs exist over a small range of $\theta, 0 \leqslant \theta \leqslant \theta_{\max }$, where $\theta_{\max }$ is a function of $\phi_{p B}$. Note that $\bar{\phi}_{3}-\phi_{p B}$ is almost independent of $\phi_{p B}$ for small $\theta\left(<7^{\circ}\right)$. Also note that Eq. (13) is again satisfied at $\theta=0$.

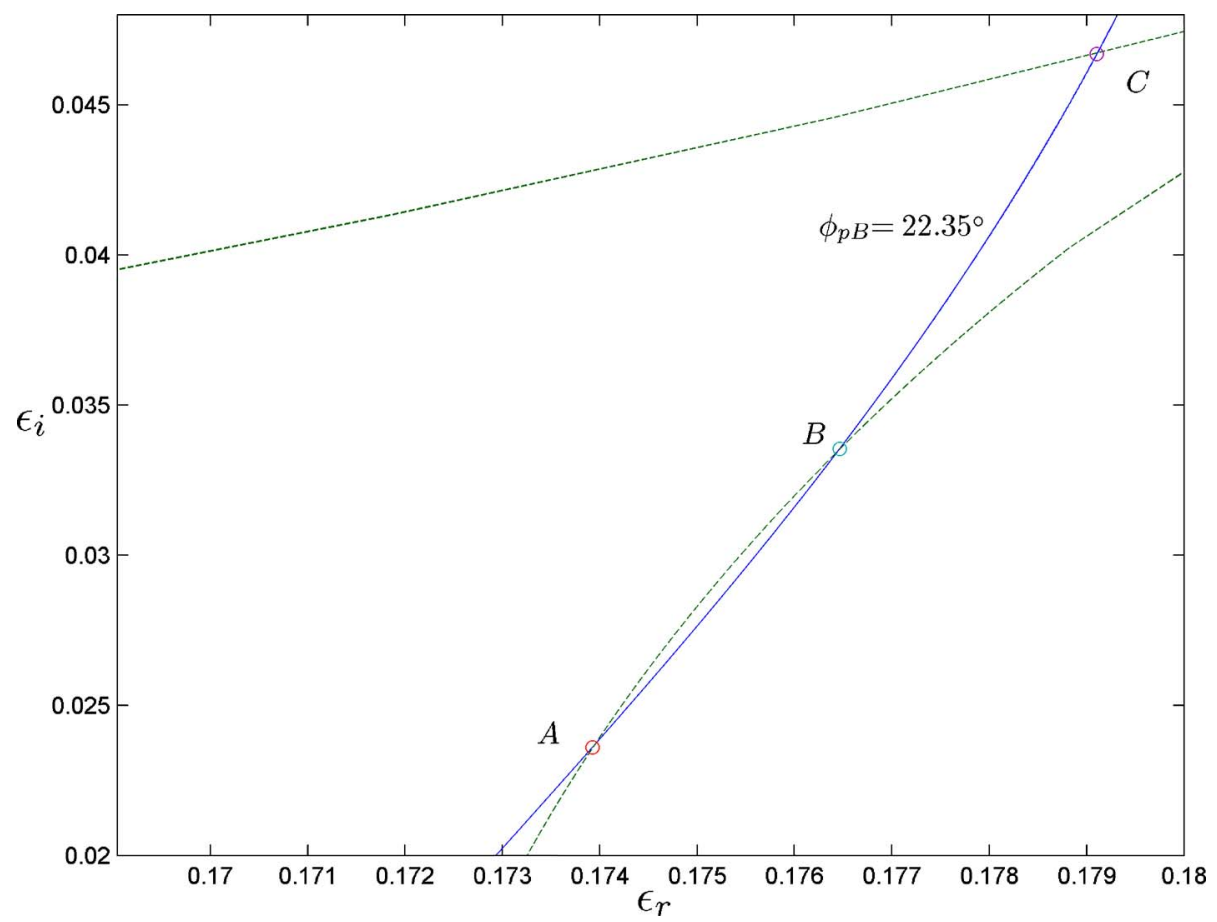

Fig. 7. (Color online) CPBAC for $\phi_{p B}=22.35^{\circ}$. This curve intersects the boundary of the domain of MPAs at three points, $A, B$, and $C$, where $\theta_{A}=7.730^{\circ}, \theta_{B}=10.763^{\circ}, \theta_{C}=14.614^{\circ}$. 


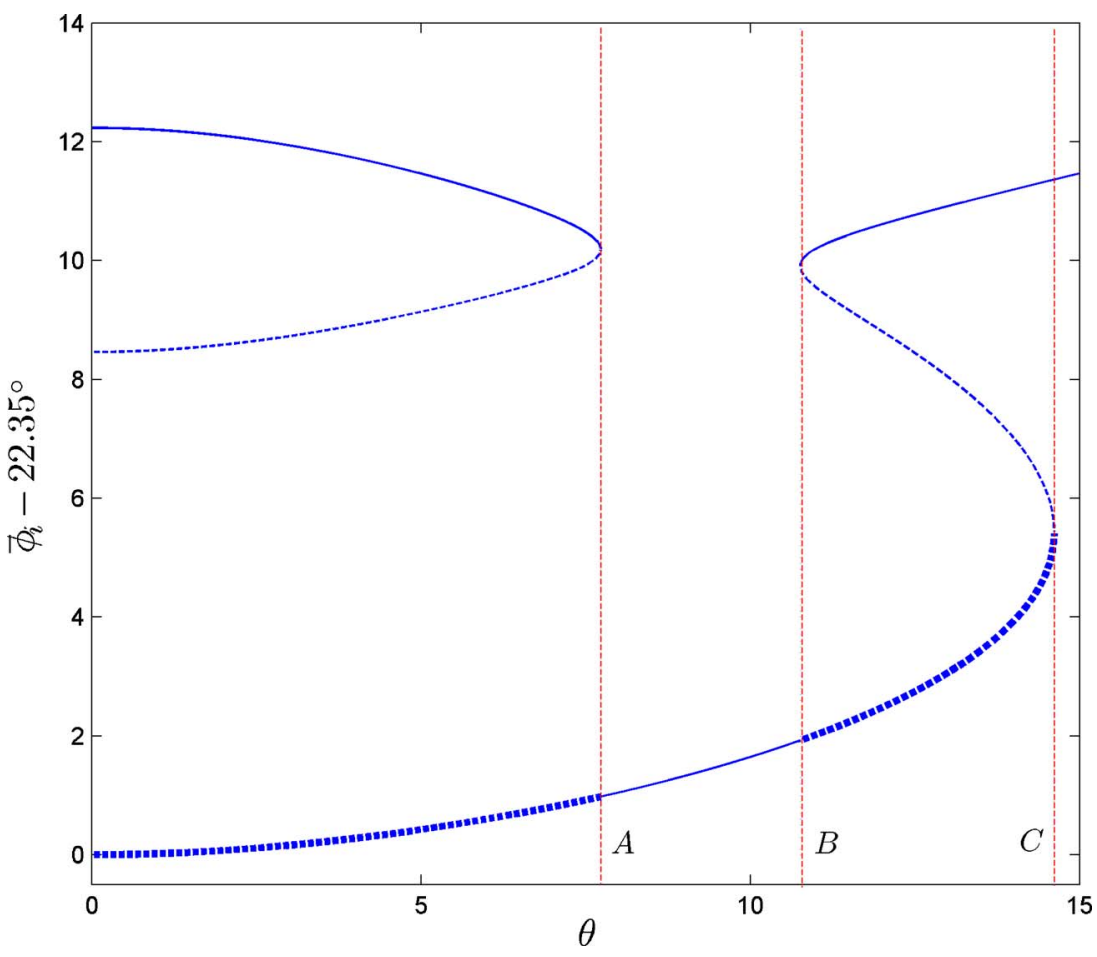

Fig. 8. (Color online) Multiple solution branches of the difference function $\bar{\phi}_{i}-\phi_{p B}, i=1,2,3$, plotted versus the angle $\theta$ of complex $\varepsilon$ when $\phi_{p B}=22.35^{\circ}$. For this PBA, MPAs exist for $0 \leqslant \theta \leqslant \theta_{A}$ and $\theta_{B} \leqslant \theta \leqslant \theta_{C}$, whereas one PA appears when $\theta_{A}<\theta<\theta_{B}$ and $\theta>\theta_{C}$.

More complex behavior is encountered in a very narrow range of the $\mathrm{PBA}, 22.339^{\circ}<\phi_{p B}<22.5^{\circ}$ Figure 7 shows the CPBAC for $\phi_{p B}=22.35^{\circ}$. This curve intersects the boundary of the region of MPAs at three points $A, B$, and $C$ where $\theta_{A}=7.730^{\circ}, \theta_{B}=10.763^{\circ}, \theta_{C}=14.614^{\circ}$.

Figure 8 shows $\bar{\phi}_{i}-\phi_{p B}, i=1,2,3$, as functions of $\theta$ when $\phi_{p B}=22.35^{\circ}$. For this PBA, MPAs exist for $0 \leqslant \theta$ $\leqslant \theta_{A}$ and $\theta_{B} \leqslant \theta \leqslant \theta_{C}$, whereas one PA appears when $\theta_{A}$ $<\theta<\theta_{B}$ and $\theta>\theta_{C}$.

Finally, Fig. 9 shows a composite plot of multiple solution branches of the difference function $\bar{\phi}_{i}-\phi_{p B}, i=1,2,3$, versus $\theta$ for $\phi_{p B}=21^{\circ}, 22^{\circ}, 22.3^{\circ}, 22.35^{\circ}, 22.5^{\circ}$, and

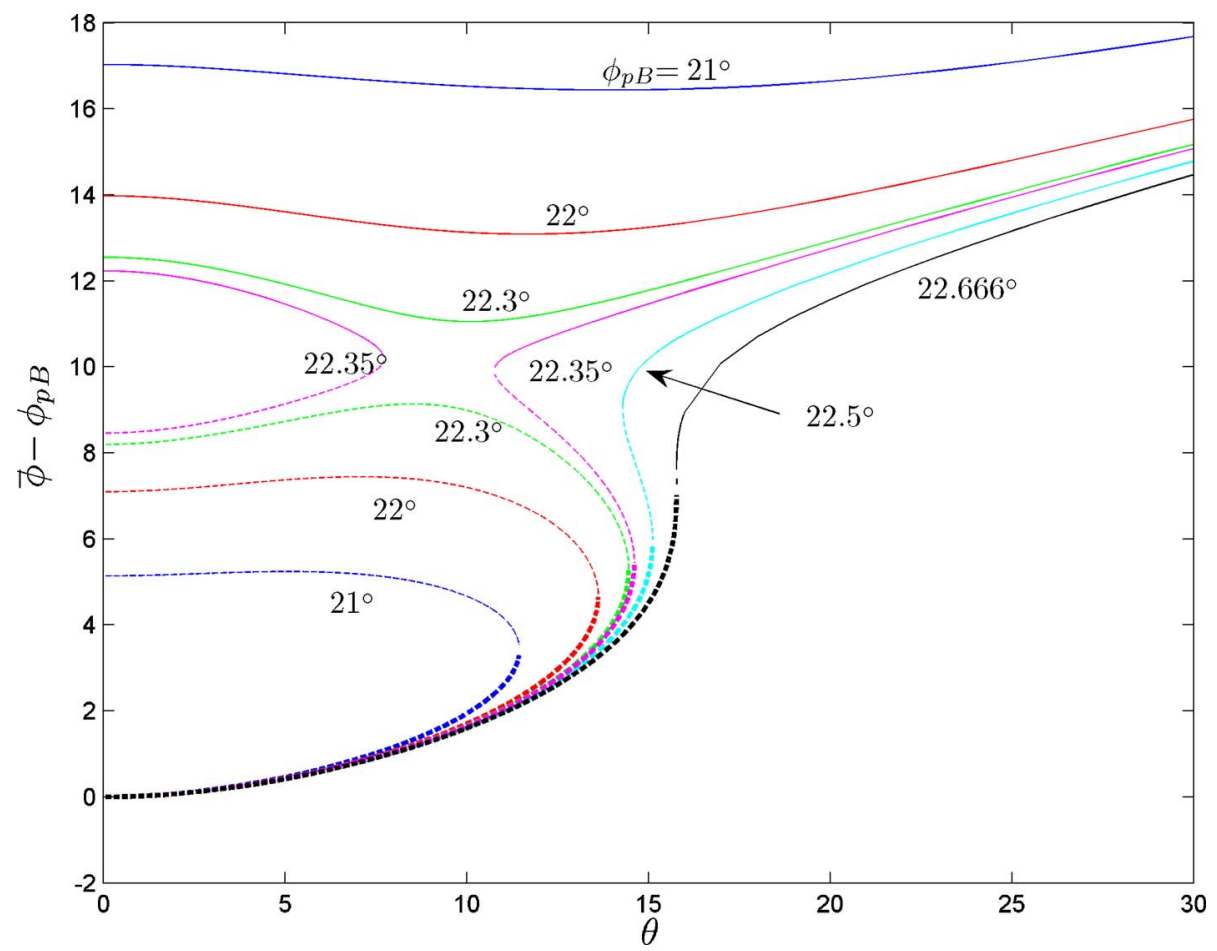

Fig. 9. (Color online) Composite plot of multiple solution branches of the difference functions $\bar{\phi}_{i}-\phi_{p B}, i=1,2,3$, for $\phi_{p B}=21^{\circ}, 22^{\circ}, 22.3^{\circ}$, $22.35^{\circ}, 22.5^{\circ}$ and $22.666^{\circ}$ in the domain of MPAs. 
$22.666^{\circ}$ in the domain of MPA. As in Fig. 6, for each $\phi_{p B}$ the solid, thin-dashed, and thick-dashed curves correspond to $\bar{\phi}_{1}>\bar{\phi}_{2}>\bar{\phi}_{3}$. Again, notice that $\bar{\phi}_{3}-\phi_{p B}$ is almost independent of $\phi_{p B}$ for small $\theta\left(<7^{\circ}\right)$.

\section{SUMMARY}

The main conclusions of this work are summarized below:

(1) Whereas there is only one unique pseudo-Brewster angle $\phi_{p B}$ that characterizes a given dielectric-conductor interface, one, two, or three principal angles $\bar{\phi}_{i}>\phi_{p B}, i$ $=1,2,3$, may exist for the same complex $\varepsilon$.

(2) For a fixed $\phi_{p B}$ there is a spread of each of the three possible associated principal angles $\bar{\phi}_{i}, i=1,2,3$.

(3) Only one principal angle $\bar{\phi}_{1}$ exists per each complex $\varepsilon$ if $\phi_{p B}>22.666^{\circ}$.

(4) The maximum difference $\left(\bar{\phi}-\phi_{p B}\right)_{\max }$ for a given $\phi_{p B}$ occurs when $\varepsilon$ becomes real negative and is determined by Eqs. (14) and (15). $\left(\bar{\phi}-\phi_{p B}\right)_{\max } \rightarrow 45^{\circ}$ and 0 in the limit as $\phi_{p B} \rightarrow 0$ and $90^{\circ}$, respectively.

(5) For $\phi_{p B} \geqslant 85^{\circ}$, we find that $\left(\bar{\phi}-\phi_{p B}\right)_{\max }<0.1^{\circ}$.

(6) Complex behavior of the difference function $\bar{\phi}_{i}$ $-\phi_{p B}, i=1,2,3$, is encountered in the domain of fractional optical constants as is illustrated by Figs. 6 and 9 .

\section{REFERENCES}

1. M. Born and E. Wolf, Principles of Optics (Cambridge U. Press, 1999).

2. R. M. A. Azzam and N. M. Bashara, Ellipsometry and Polarized Light (North-Holland, 1987).
3. J. Lekner, Theory of Reflection (Martinus, Nijhoff, 1987).

4. S. P. F. Humphreys-Owen, "Comparison of reflection methods for measuring optical constants without polarimetric analysis, and proposal for new methods based on the Brewster angle," Proc. Phys. Soc. London 77, 949-957 (1961).

5. H. B. Holl, "Specular reflection and characteristics of reflected light," J. Opt. Soc. Am. 57, 683-690 (1967).

6. G. P. Ohman, "The pseudo-Brewster angle," IEEE Trans. Antennas Propag. AP-25, 903-904 (1977).

7. R. M. A. Azzam, "Maximum minimum reflectance of parallel-polarized light at interfaces between transparent and absorbing media," J. Opt. Soc. Am. 73, 959-962 (1983).

8. S. Y. Kim and K. Vedam, "Analytic solution of the pseudoBrewster angle,” J. Opt. Soc. Am. A 3, 1772-1773 (1986).

9. L. R. Hooper, J. R. Sambles, and A. P. Bassom, "The transverse magnetic reflectivity minimum of metals," Opt. Express 16, 7580-7586 (2008).

10. R. M. A. Azzam, "Contours of constant principal angle and constant principal azimuth in the complex $\varepsilon$ plane," J. Opt. Soc. Am. 71, 1523-1528 (1981).

11. E. D. Palik, ed., Handbook of Optical Constants of Solids (Academic, 1985).

12. D. L. Windt, W. C. Cash, Jr., M. Scott, P. Arendt, B. Newman, R. F. Fisher, A. B. Swartzlander, P. Z. Takacs, and J. M. Pinneo, "Optical constants for thin films of C, diamond, $\mathrm{Al}, \mathrm{Si}$, and CVD SiC from $24 \AA$ to $1216 \AA$," Appl. Opt. 27, 279-295 (1988).

13. R. M. A. Azzam, "Differential reflection phase shift under conditions of attenuated internal reflection," J. Opt. Soc. Am. A 16, 1700-1702 (1999).

14. R. M. A. Azzam and E. Ugbo, "Contours of constant pseudoBrewster angle in the complex $\varepsilon$ plane and an analytical method for the determination of optical constants," Appl. Opt. 28, 5222-5228 (1989).

15. R. M. A. Azzam and E. Ugbo, "Angular range for reflection of $p$-polarized light at the surface of an absorbing medium with reflectance below that at normal incidence," J. Opt. Soc. Am. A 19, 112-115 (2002). 